\title{
Abordaje odontopedátrico de un paciente con Displasia Ectodérmica
}

Juan Diego Mejía Roldan ${ }^{1}$

Luz Beatriz Vera Uribe ${ }^{2}$

\section{Resumen}

La Displasia Ectodérmica hace parte un grupo de afecciones o síndromes, que tienen en común defectos en el desarrollo normal de dos o más tejidos derivados del ectodermo, en los cuales hay un desarrollo anormal de la piel, dientes, pelo, uñas o glándulas sudoríparas. Presenta oligodoncia, dientes en clavija y reab- sorción de procesos alveolares, se le realizó un tratamiento integral odontológico, consiguiendo una estética, funcionalidad y adaptación excelente así como una considerable mejora de la autoestima.

Palabras clave: Displasia Ectodérmica, oligodoncia, tratamiento dental, rehabilitación oral, manifestaciones orales.

Relato de caso

\section{Tratamento odontológico de criança com Displasia Ectodérmica}

\section{Resumo}

Displasia Ectodérmica é parte de um grupo de condições ou síndromes, que têm em comum, defeitos comuns no desenvolvimento normal de dois ou mais tecidos derivados de ectoderma, podendo haver desenvolvimento anormal da pele, dentes, unhas e cabelo, glândulas sudoríparas. Este artigo descreve um caos de criança com 4 anos de idade com displasia do ectoderma, apresentando oligodontia, dentes conóides e reabsorção de processos alveolares. Foi realizado tratamento odontológico integral, alcançando resultado estético, funcionalidade e excelente adaptação, bem como uma melhoria significativa na auto-estima.

Palavras-chave: Displasia ectodérmica, oligodontia, tratamento dental, reabilitação oral, manifestações orais.

\section{Case report}

\section{Dental treatment for a pedatric patient with Ectodermal Dysplasia}

\author{
Abstract \\ mon in the ordinary development of two or more \\ Ectodermal Dysplasias forms are a group of di- \\ tissues derived from ectoderm are common in \\ seases or syndromes in which defects are com- \\ which there is abnormal development of the skin, \\ ${ }^{1}$ Odontopediatria Universidad CES Medellin, Colombia \\ ${ }^{2}$ Residente de tercer año del posgrado de odontopediatría y ortodoncia preventiva. Universidad CES Medellín, Colombia
}


teeth, hair, nails and sweat glands may be observed. This article describes, the case of a 4 year old child with Ectodermal Dysplasia who presented oligodontia, peg teeth and resorption of alveolar processes. Comprehensive dental treatment was is exposed, To Whom Realized is an integrated dental treatment, Achieving aesthetics, excellent functionality and adaptation as well as a significant improvement in his self-esteem.

Keywords: Ectodermal Dysplasia, oligodontia, dental treatment, oral rehabilitation, Oral manifestations

\section{Introducción}

La displasia ectodérmica conforma un grupo de afecciones o síndromes que tienen en común defectos en el normal desarrollo de dos o más tejidos derivados del ectodermo, en los cuales hay un desarrollo anormal de la piel, dientes, pelo, uñas o glándulas sudoríparas. (1)

Es un desorden que no se presenta con mucha frecuencia. Esta varía entre 1:10.000 y 1:100.000 niños nacidos vivos y es más frecuente en los hombres. En la mayoría de los casos es de herencia autosómica recesiva, pero puede ser también autosómica dominante o ligada al cromosoma X. (2)

Muchos defectos en los genes pueden generar una Displasia Ectodérmica por lo cual existen muchos tipos de displasias, observándose diferentes síndromes, dando como resultado hasta 154 formas diferentes de Displasia Ectodérmica y 11 subgrupos. Los tipos que se observan más frecuentemente son: los hipohidrótico, anhidróticos y los hidróticos. (1)

El tipo hipohidrótico-anhidrótico, síndrome de Christ- Siemens-Touraine, fue descrito en 1848 por Thurman y se caracteriza por la tríada hipotricosis (anomalías en piel, pelo y uñas), hipodoncia o anodoncia e hipohidrosis (ausencia parcial o total de glándulas sudoríparas ecrinas) y algunos rasgos como: abombamiento de la frente, nariz en silla de montar, labios evertidos. (3)

El tipo hidrótico es definido en 1929 por Clouston y se distingue por hipotricosis, distrofia ungueal e hiperqueratosis de palmas de las manos y plantas de los pies.(3)

La hipodoncia es la falta de 1 o más dientes $(<6)$; la oligodoncia es la ausencia de 6 o más dientes; y la anodoncia total es la ausencia de la dentición; estas son de gran interés a la hora de diagnosticar las displasias (4) La oligodoncia se encuentra asociada con más frecuencia a factores hereditarios de tipo dominante, poligénico y ligado al cromosoma X. También es muy frecuente en estos pacientes el hallazgo de anomalías en la forma y tamaño de los dientes, y alteración en la erupción. $(5,6)$

El diagnóstico diferencial de las Displasias Ectodérmicas está basado principalmente en la historia clínica, se debe analizar la patología, hacer diagnóstico prenatal de forma invasiva (biopsia de piel o un estudio genético) o no invasiva mediante ultrasonografía tridimensional identificando las características faciales a partir de la semana treinta de gestación.(3)

El pronóstico, en general, es bueno, salvo los casos con hipo o anhidrosis, que alcanzan tasas de mortalidad del $30 \%$ en la primera infancia, fundamentalmente, debido a infecciones respiratorias dada la ausencia de glándulas mucosas en el aparato respiratorio.

En odontopediatría se usan diferentes tratamientos para darles a estos pacientes una solu- 
ción estética y funcional a muy temprana edad. El tratamiento odontológico debe ser de acuerdo a la edad, a la severidad del caso y al desarrollo dental.

Las formas plásticas son una buena opción para mejorar la anomalía en la forma dental en la dentición decidua, son restauraciones adhesivas con gran apariencia estética y buen control de la hemorragia. La retención depende de la cantidad de estructura dentaria con la que se cuente y las fuerzas a la que está expuesta. Las ventajas de restaurar con las formas plásticas es la alta estética que se logra, el menor tiempo de trabajo, requiere remoción de poco tejido dental, se puede obtener retención extra adhiriéndose al esmalte y dentina remanente.(7)

El tratamiento de rehabilitación es importante por razones fisiológicas, emocionales y psicosociales, el inicio de este tratamiento debe ser lo más temprano posible para limitar la reabsorción de los procesos alveolares.

El objetivo de este artículo es reportar un caso clínico, de un paciente de 4 años con diagnóstico de Displasia Ectodérmica, el cual presenta múltiples afecciones a nivel dental y es tratado en el servicio de odontopediatría, se sustenta con evidencia en la literatura, y se intenta responder la siguiente pregunta, cuales son los efectos fisiológicos y sicológicos de un tratamiento odontopediátrico integral en un paciente con Displasia Ectodérmica?

\section{Caso clínico}

Paciente de 4 años de edad de sexo masculino que asiste a consulta de odontopediatría por primera vez a la Universidad CES en el 2013, en compañía de su madre remitidos por el odontopediatra de su empresa prestadora de salud.
Motivo de consulta: "tiene los dientes puntiagudos y quiero que se los limen, además va a entrar a la guardería y no quiero que los niños lo vean sin dientes. El se siente mal por no tener dientes" relata la madre.

ENFERMEDAD ACTUAL: Paciente asintomático, el cuál consulta para tratamiento odontopediatríco por anomalía en la forma de los dientes.

Se realizó historia clínica completa, en los antecedentes médicos presenta antecedente de Bronquitis, Rinitis y Dermatitis de contacto y parto por cesaría.

ANTECEDENTES FAMILIARES: Su madre relata que en ella, un tío y el abuelo materno del niño, presentan ausencias y forma cónica de los dientes.

EXAMEN EXTRAORAL: Paciente con tipo craneal mesocéfalico, tipo facial mesoprosopo con cuerpo ectomorfo, pelo escaso y fino, con desarrollo psicomotor y crecimiento normal.

EXAMEN FACIAL: Tipo facial ortognático, tercios 50 - 48 - 40 con tercio inferior disminuido, presenta competencia labial, frente amplia, puente nasal aplanado, nariz pequeña con dorso recto, orejas bajas, mentón efectivo, con arcos superciliares poco prominentes, malar poco expresivo, surco frontonasal obtuso, ángulo naso labial obtuso, surco mentolabial marcado, perfil recto, línea media facial desviada hacia la izquierda por deflexión mandibular, sonrisa dental, filtrum centrado. (Figura 1).

EXAMEN INTRAORAL: Se observa atrofia ósea de los procesos alveolares tanto en el maxilar como en mandíbula y numerosos espacios edéntulos, presenta anomalía en la forma y en el número de dientes (figura 1); se observa oligodoncia y dientes en forma de clavija, periodon- 
talmente se observa inflamación generalizada asociada a biopelícula dental, pulpar sano, tipo de dentición decidua, riesgo bajo de caries. Se

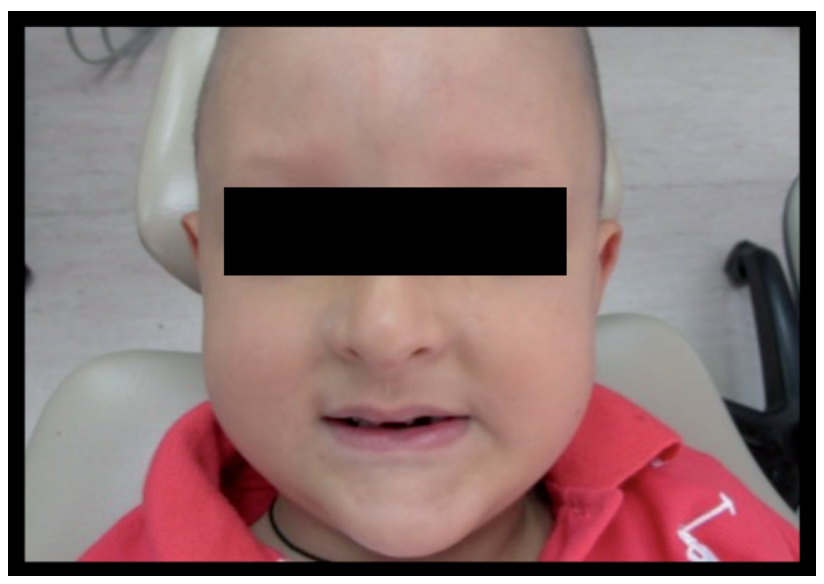

Figura 1. Primera cita.

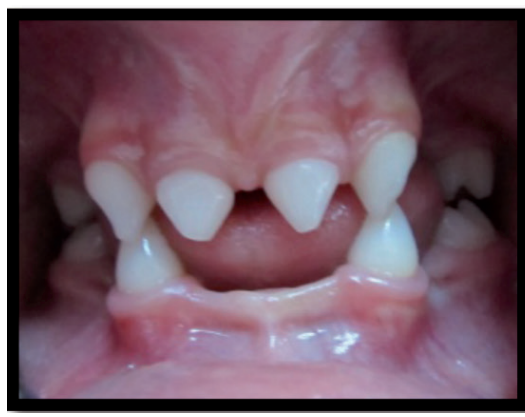

Figura 2. Oclusal de frente.

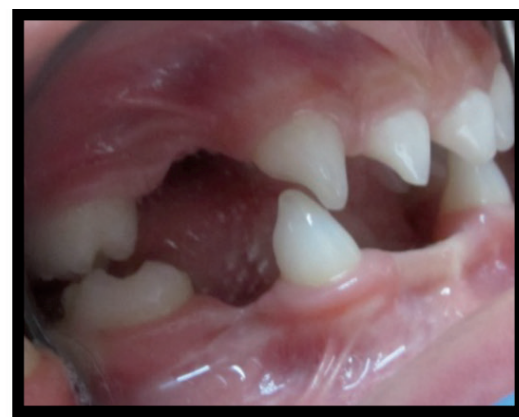

Figura 3. Lateral izquierda. observó una relación molar decidua de escalón mesial bilateral, presenta contacto prematuro entre 63-73 generando una deflexión mandibular. (Figura 2-3-4)

Se observa forma cuadrada en los arcos dentarios superior e inferior. (Figura 5-6)

\section{Ayudas diagnósticas}

RADIOGRAFIA PANORAMICA: Presenta dentición decidua con numerosas agenesias (oligodoncia), se observa retraso en la erupción y formas dentarias anómalas. (Figura 7)

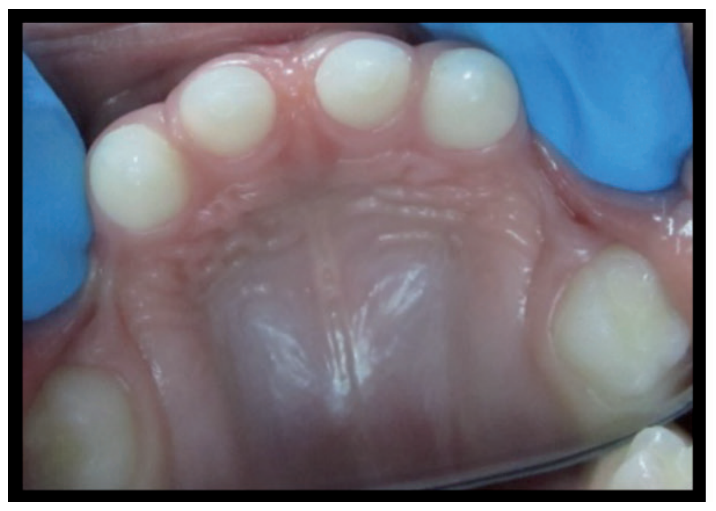

Figura 5. Oclusal superior.

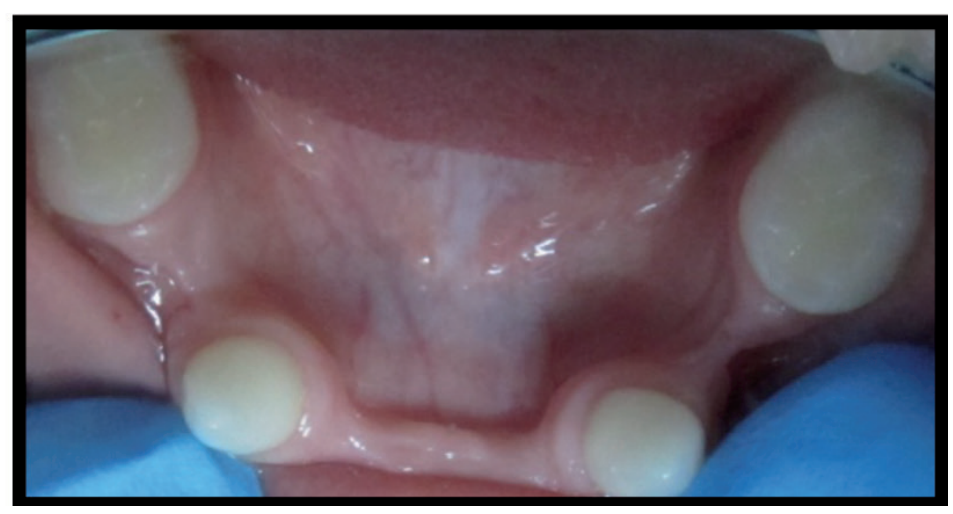

Figura 6. Oclusal inferior.

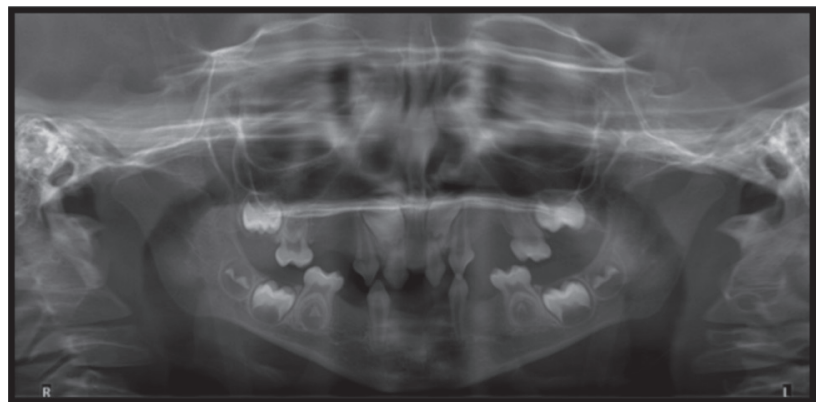

Fórmula dental con ausencias dentales

\begin{tabular}{rrr|ccc}
16 & & 11 & 21 & \multicolumn{2}{c}{26} \\
55 & 53 & 51 & 61 & 63 & 65 \\
\hline 85 & 83 & & & 73 & 75 \\
474645 & & & & & 353637
\end{tabular}

Figura 7. Panorámica. 


\section{Diagnósticos}

SISTÉMICO: Bronquitis, Rinitis y Dermatitis de contacto controlado, diagnóstico presuntivo de Displasia Ectodérmica.

ESTOMATOLÓGICO: Diagnóstico presuntivo de Displasia Ectodérmica.

FACIAL: Perfil recto, tipo facial ortognático, frente amplia, orejas bajas, tercio medio disminuido, desviación mandibular a la izquierda.

PERIODONTAL: Gingivitis generalizada asociada a biopelícula.

DENTAL: Oligodoncia :

- Deciduos: 54-52-62-64- 74-72-71-81-82-84.

- Permanentes:17-15-14-13-12-22-23-24-2527-34-33-32-31-41-42-43-44.

- Anomalía de forma dental: Dientes en clavija 53-51-61-63-73-83.

PULPAR: Sano.

OCLUSAL: Maloclusión clase III bilateral.

FUNCIONAL: Deflexión mandibular a la izquierda por contacto prematuro entre 63 y 73 .

OBJETIVOS DEL TRATAMIENTO: Dar solución aceptable a un caso complejo debido a la corta edad del niño y la situación emocional materna, agobiada por la numerosa ausencia de dientes en zonas tan comprometidas estéticamente. Se busco restablecer la función, mejorar la estabilidad oclusal, la dimensión vertical y la desviación mandibular.

Se inició el tratamiento de rehabilitación lo antes posible, para limitar la reabsorción de los procesos alveolares, minimizar el impacto psicológico y emocional por la ausencia de dientes, mejorar la forma de los dientes en clavija, confirmar diagnóstico sistémico de Displasia Ectodérmica y poder guiar a la familia a una asesoría genética.

\section{Plan de tratamiento 2013}

PERIODO HIGIENICO: Motivación e instrucción en higiene bucal y profilaxis. Remisión a genetista y a pediatría.

PERIODO CORRECTIVO INICIAL: Formas plásticas para mejorar forma en clavija de los dientes: 53-51-61-63-73-83, instalación del flipper mantenedor de espacio fijo para rehabilitar zonas edéntulas inferiores con bandas en 75 y 85. Se decide poner aparatología fija por la edad del paciente y el difícil manejo.

PERIODO CORRECTIVO FINAL: Reevaluación y control del flipper mantenedor de espacio fijo y asesoría para rehabilitaciones posteriores y acompañamiento a la familia en el crecimiento y desarrollo del niño.

Se realizó interconsulta con patólogo oral el cual por los signos clínicos confirma el diagnóstico de displasia ectodérmica, y recomienda remitir a genetista para que defina qué tipo de displasia presenta.

En abril del 2013 se realizó formas plásticas superiores 53-51-61-63 e inferiores 73 y 83 con el objetivo de darle una forma más anatómica a los dientes. (Figura 8-9)

En mayo del 2013 se adaptaron bandas inferiores en 75 y 85 para fabricación del flipper, mantenedor de espacio fijo, se toma impresión en alginato para su fabricación, se le envía al laboratorio con una foto del paciente y con el color de los dientes. (Figura 10) 


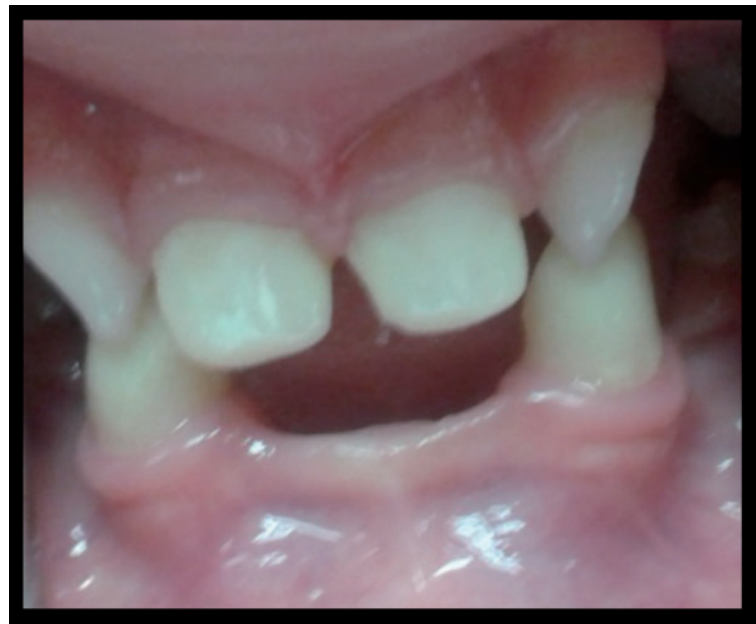

Figura 8. Formas plásticas 51-61.

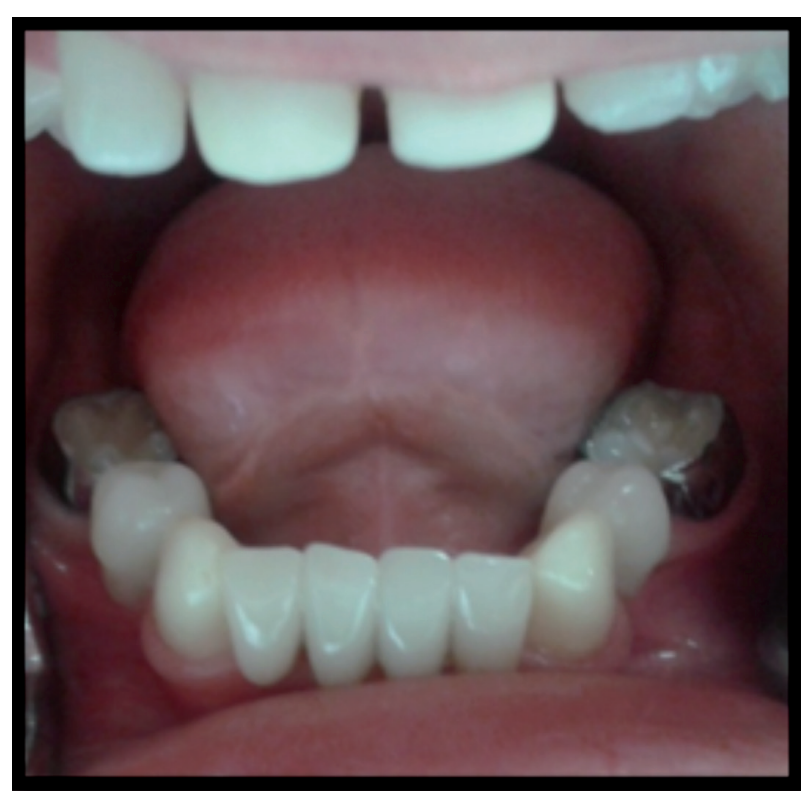

En junio se cementa flipper mantenedor de espacio fijo y se logra una adecuada adaptación.

El paciente es citado a control cada 3 meses para control de aparatología e higiene oral.

La madre relata que el niño sicológicamente se observa que se le facilita mas la socialización en la guardería, la mama se encuentra muy satisfecha con el resultado del tratamiento y está actualmente en la asesoría genética, mejoro en el habla y la masticación.

Figura 10. Flipper.Mantenedor de espacio fijo.

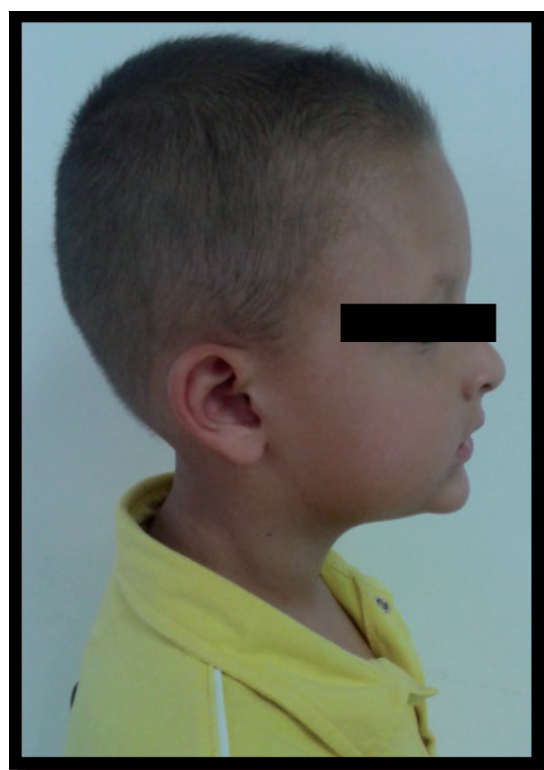

Figura 11. Foto perfil.

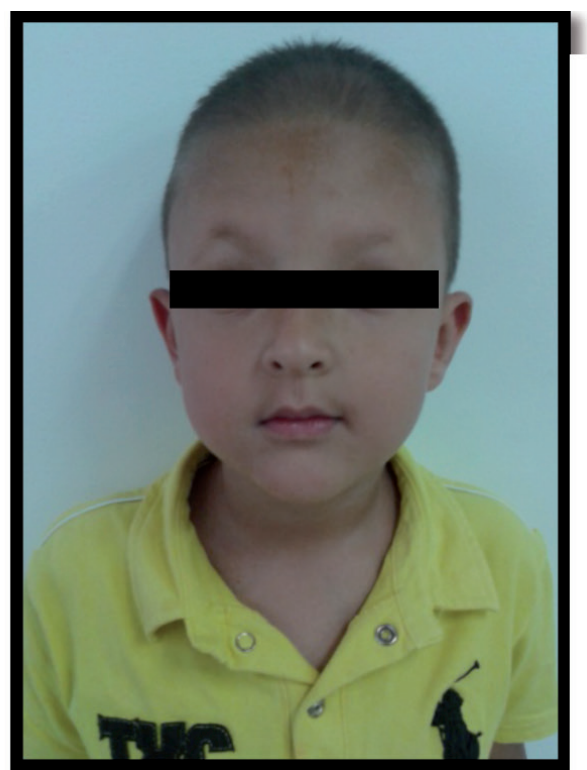

Figura 12. Foto de frente.

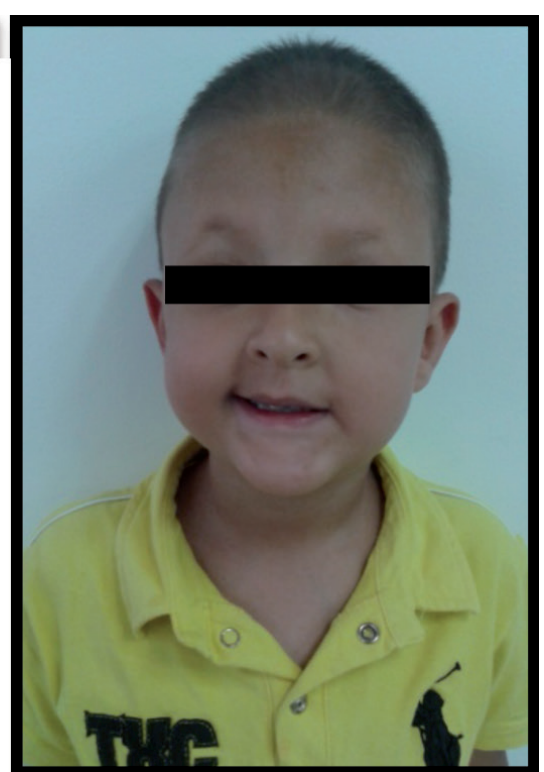

Figura 13. Foto sonrisa. 
La madre autoriza el tratamiento y la publicación del caso.

\section{Resultados}

A nivel facial: el paciente presenta más estabilidad oclusal logrando eliminar deflexión mandibular y eliminando asimetría facial. Se logró aumento de la dimensión vertical.

A nivel dental se logra una forma dental estética y funcional, ya que con las formas plásticas se les dio a los dientes la forma anatómica normal. Se logra con el flipper mantenedor de espacio fijo remplazar dientes faltantes inferiores protegiendo el reborde alveolar, devolviendo la estética y la función.

A nivel funcional se eliminó deflexión mandibular al restablecer la oclusión, mejoramiento en la fonación, masticación, habla y autoestima del paciente pediátrico.

\section{Discusión}

Para poder sustentar con evidencia científica este reporte de caso se realiza una pregunta PICO; cuales son los efectos fisiológicos y sicológicos de un tratamiento odontopediátrico integral en un paciente con Displasia Ectodérmica?

Se realizó una revisión de literatura mediante la búsqueda en la base de datos MEDLINE (vía PubMed, Cochrane (Biblioteca Cochrane Plus), LILACS (search vía virtual Health Library), Google Scholar. La revisión se realizó del período comprendido entre 1980 a 2014; se utilizó el Medical Subject Headings (MeSH). Se incluyeron todos los estudios sobre los efectos fisiológicos y sicológicos de un tratamiento odontopediátrico integral en un paciente con Displasia Ectodérmica? Se usaron los siguientes términos Mesh: "Ectodermal Dysplasia", "children oral rehabilitation", “Oral Manifestations".

Tabla 1. Resultados de la búsqueda según términos MESH.

\begin{tabular}{|c|c|}
\hline MESH TERMS & $\begin{array}{c}\text { RESULTADOS } \\
\text { artículos }\end{array}$ \\
\hline "Ectodermal Dysplasia" AND (clinical trial) & 7 \\
\hline "Ectodermal Dysplasia” AND “children oral rehabilitation” & 70 \\
\hline "Ectodermal Dysplasia" AND "oral Manifestations" & 45 \\
\hline
\end{tabular}

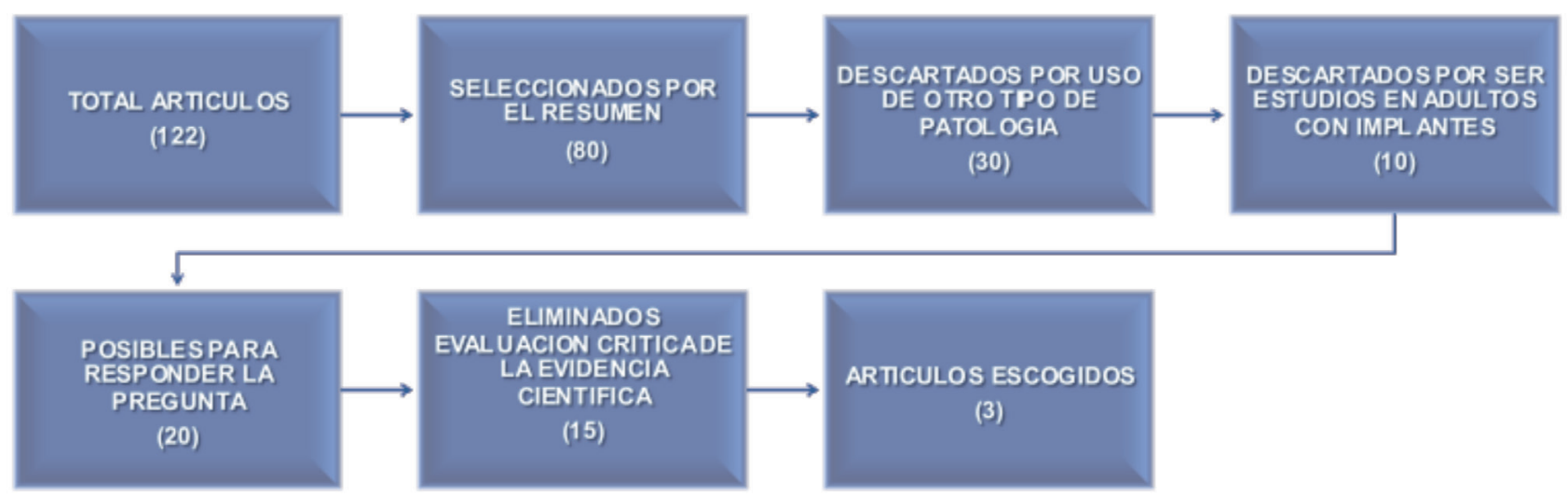

Esquema 1. Artículos encontrados en la búsqueda. 
Tabla 2. Resumen artículos seleccionados.

\begin{tabular}{|c|c|c|c|c|}
\hline $\begin{array}{c}\text { TITULO / } \\
\text { AUTOR } \\
\text { AÑO }\end{array}$ & OBJETIVO & REPORTE DEL CASO & TRATAMIENTO & CONCLUSIONES \\
\hline $\begin{array}{c}\text { A simple } \\
\text { prosthetic res- } \\
\text { torative solu- } \\
\text { tionof a single } \\
\text { peg-shaped } \\
\text { upper central- } \\
\text { primary inci- } \\
\text { sor in a case } \\
\text { ofectodermal } \\
\text { displasia } \\
\text { ANCA MARIA } \\
\text { RĂDUCANU, } \\
\text { MIHAELA } \\
\text { PĂUNA, I. V. } \\
\text { FERARU } \\
\text { 2010 (8) }\end{array}$ & $\begin{array}{l}\text { Informe clínico que } \\
\text { describe la rehabi- } \\
\text { litación oral de un } \\
\text { niño de } 7 \text { años de } \\
\text { edad con diagnós- } \\
\text { tico de Displasia } \\
\text { Ectodérmica hipohi- } \\
\text { drótica y anodoncia. } \\
\text { El tratamiento se } \\
\text { centra en mejorar } \\
\text { sus necesidades } \\
\text { estéticas y funciones } \\
\text { orales e incluyó la } \\
\text { fabricación de pró- } \\
\text { tesis removibles y } \\
\text { de una corona de } \\
\text { acrílico en el único } \\
\text { diente existente en } \\
\text { la boca. }\end{array}$ & $\begin{array}{c}\text { Rehabilitación oral de un } \\
\text { varón de } 7 \text { años de edad } \\
\text { con Displasia Ectodérmica } \\
\text { recesiva ligada al cromoso- } \\
\text { ma X anhidrótica. } \\
\text { Madre con DE. } \\
\text { EXAMEN CLINICO FA- } \\
\text { CIAL ORAL: frente y } \\
\text { mentón prominente, el pelo } \\
\text { frágil y fino, una nariz en } \\
\text { forma de silla de montar, } \\
\text { un surco nasolabial obtuso, } \\
\text { un labio superior delgado, } \\
\text { un labio inferior evertido, } \\
\text { y orejas bajas, tercio facial } \\
\text { inferior reducido y cejas y } \\
\text { pestañas escasas } \\
\text { Presenta un diente en forma } \\
\text { de cono maxilar, perdida de } \\
\text { la dimensión vertical, au- } \\
\text { sencia de procesos alveola- } \\
\text { res y un desarrollo anormal } \\
\text { de rebordes alveolares }\end{array}$ & $\begin{array}{c}\text { Los objetivos del tra- } \\
\text { tamiento oral fueron } \\
\text { mejorar la estética y las } \\
\text { funciones orales espe- } \\
\text { cialmente la mastica- } \\
\text { ción, permitiendo el } \\
\text { desarrollo psicológico } \\
\text { normal. } \\
\text { Tratamiento protésico } \\
\text { convencional. Remo- } \\
\text { vible maxilar y mandi- } \\
\text { bular completa. }\end{array}$ & $\begin{array}{l}\text { Las condiciones psico- } \\
\text { lógicas, el perfil facial, } \\
\text { así como las funciones } \\
\text { y estética de la paciente } \\
\text { mejoraron. Se obtuvie- } \\
\text { ron mejoras notables } \\
\text { en el habla y el estado } \\
\text { nutricional del paciente } \\
\text { joven. }\end{array}$ \\
\hline $\begin{array}{c}\text { Alternative } \\
\text { oral rehabilita- } \\
\text { tion of children } \\
\text { with hypodon- } \\
\text { tia and conical } \\
\text { tooth shape: A } \\
\text { clinical report } \\
\text { Vivien Thiemy } \\
\text { Sakai, DDS1/ } \\
\text { Thais Marchini } \\
\text { Oliveira. } \\
2006 \text { (9) }\end{array}$ & $\begin{array}{l}\text { En este artículo se } \\
\text { informa de una } \\
\text { rehabilitación alter- } \\
\text { nativa que implica } \\
\text { la remodelación de } \\
\text { dientes cónicos con } \\
\text { resina compuesta } \\
\text { y el uso de prótesis } \\
\text { removibles. }\end{array}$ & $\begin{array}{l}\text { Un niño de } 4 \text { años de edad } \\
\text { con hipodoncia y la forma } \\
\text { de los dientes cónicos } \\
\text { Hipodoncia, disminución } \\
\text { de dimensión vertical, inci- } \\
\text { sivos centrales superiores } \\
\text { muy separados, y xerosto- } \\
\text { mía. Todos los dientes ante- } \\
\text { riores eran de forma cónica. } \\
\text { Agenesia de varios dientes } \\
\text { permanentes }\end{array}$ & $\begin{array}{l}\text { El plan de tratamien- } \\
\text { to construcción de } \\
\text { prótesis parciales } \\
\text { removibles después } \\
\text { de la remodelación de } \\
\text { los dientes anteriores } \\
\text { superiores con forma } \\
\text { cónicas y posterior } \\
\text { restauración con resina } \\
\text { compuesta }\end{array}$ & $\begin{array}{l}\text { Remodelación de dien- } \\
\text { tes cónicos con resina } \\
\text { compuesta y el uso de } \\
\text { prótesis removibles, } \\
\text { puede ser de ayuda } \\
\text { para la rehabilitación } \\
\text { de pacientes con hipo- } \\
\text { doncia y la forma cóni- } \\
\text { ca del diente durante } \\
\text { los años de crecimiento } \\
\text { facial activo y puede } \\
\text { mejorar la calidad de } \\
\text { vida de estos niños }\end{array}$ \\
\hline
\end{tabular}




\begin{tabular}{|c|c|c|c|c|}
\hline $\begin{array}{l}\text { Hypohidrotic } \\
\text { ectodermal } \\
\text { dysplasia with } \\
\text { true anodontia } \\
\text { of the primary } \\
\text { dentition } \\
\text { Aydan } \\
\text { Açıkgöz, Oya } \\
\text { Kademoglu, }\end{array}$ & $\begin{array}{c}\text { En este informe, se } \\
\text { presenta un niño } \\
\text { con Displasia Ecto- } \\
\text { dérmica hipohidró- } \\
\text { tica tener verdadera } \\
\text { anodoncia de la } \\
\text { dentición primaria }\end{array}$ & $\begin{array}{l}\text { Un niño de } 6 \text { años de edad } \\
\text { Presentaba abombamiento } \\
\text { frontal, prominentes arcos } \\
\text { superciliares y la barbilla, } \\
\text { y una dimensión vertical } \\
\text { disminuida debido a ano- } \\
\text { doncia total, lo que le dio } \\
\text { un aspecto envejecido. } \\
\text { Ausencia total de dientes y } \\
\text { crestas alveolares delgadas, } \\
\text { paladar normal } \\
\text { La panorámica no revelo } \\
\text { los dientes primarios y } \\
\text { permanentes solo caninos } \\
\text { permanentes sin erupcionar } \\
\text { bilateralmente en el maxilar } \\
\text { superior }\end{array}$ & $\begin{array}{l}\text { Rehabilitación proté- } \\
\text { sica total superior e } \\
\text { inferior } \\
\text { Se permitió erupción } \\
\text { de los caninos y se } \\
\text { realizó resina para } \\
\text { mejorar su estética. }\end{array}$ & $\begin{array}{l}\text { El equipo dental debe } \\
\text { ser consciente de los } \\
\text { signos y síntomas de la } \\
\text { displasia ectodérmica } \\
\text { con el fin de propor- } \\
\text { cionar las terapias } \\
\text { correctas para las ne- } \\
\text { cesidades funcionales } \\
\text { y psicológicas de estos } \\
\text { pacientes. }\end{array}$ \\
\hline
\end{tabular}

Los resultados de la estrategia de búsqueda arrojaron un de total 122 artículos, de los cuales solo 3 fueron incluidos para sustentar el caso clínico. El nivel de evidencia es muy bajo, solo se reporta casos clínicos donde se describen diferentes formas de tratamiento y de abordaje clínico.

El paciente de este caso clínico presenta manifestaciones faciales y orales propias de esta patología; frente amplia, puente nasal aplanado, nariz pequeña, orejas bajas, pelo fino, seco e hipocrómico y oligodoncia de deciduos y permanentes.

Diversos autores han propuesto rehabilitación protésica como tratamiento de elección para este tipo de pacientes. Se recomienda rehabilitar al paciente en las primeras edades de la vida para permitir el ajuste de la dimensión vertical y una mejor interrelación entre los maxilares. Lo que permite una mejor función masticatoria, estética y desarrollo psicológico del niño.
La rehabilitación intenta mantener los procesos alveolares maxilares sin atrofia y posibilitar una vida social prácticamente normal tan importante a estas edades en el desarrollo psicológico, autoestima y de la personalidad del niño.

\section{Conclusiones}

Este caso clínico demuestra la importancia del tratamiento odontopediátrico en la rehabilitación en niños con displasia ectodérmica. Aparatología como el flipper mantenedor de espacio fijo y las formas plásticas puede ser un método reversible, estético, funcional y de bajo costo.

A nivel facial se logra eliminar deflexión mandibular y asimetría facial. También se logra aumento de la dimensión vertical.

A nivel dental se obtiene una forma dental estética y funcional, con las formas plásticas se les da a los dientes la forma anatómica normal. Se 
logra con el flipper mantenedor de espacio fijo remplazar dientes faltantes inferiores protegiendo el reborde alveolar, devolviendo la estética y la función

A nivel funcional se eliminó deflexión mandibular al restablecer la oclusión, mejoramiento en la fonación, masticación, habla y autoestima del paciente pediátrico.

Es importante lograr un acompañamiento con los padres desde edades muy tempranas y poderlos guiar en el crecimiento y desarrollo del niño.

\section{Bibliografía}

1. Pinheiro M, Freire-Maia N. Ectodermal dysplasias: a clinical classification and a causal review. Am J Med Genet.1994;53(2):153-62.

2. Lamartine J. Towards a new classification of ectodermal dysplasias. Clin Exp Dermatol. 2003;28(4):351-5.

3. Sepulveda W, Sandoval R, Carstens E, Gutierrez J, Vasquez P. Hypohidrotic ectodermal dysplasia: prenatal diagnosis by three-dimensional ultrasonography. J Ultrasound Med Off J Am Inst Ultrasound Med. 2003;22(7):731-5.

4. Bergendal B. Orodental manifestations in ectodermal dysplasia-A review. Am J Med Genet A. 2014;164(10):2465-71.

5. Tsai PF, Chiou HR, Tseng CC. Oligodontia--a case report. Quintessence Int Berl Ger 1985. 1998;29(3):191-3.

6. Hattab FN, Angmar-Månsson B. Oligodontia of the permanent dentition in two sisters with polycystic ovarian syndrome: case reports. Oral Surg Oral Med Oral Pathol Oral Radiol Endod. 1997;84(4):368-71.

7. Pigno MA, Blackman RB, Cronin RJ, Cavazos E. Prosthodontic management of ectodermal dysplasia: a review of the literature. J Prosthet Dent. 1996;76(5):541-5.

8. Răducanu A, Mihaela, i. V. Feraru. A simple prosthetic restorative solution of a single peg-shaped upper central primary incisor in a case of ectodermal displasia. Romanian Journal of Morphology and Embryology 2010, 51(2):371-374

9. Thiemy Sakai V, Marchini Oliveira T. Alternative oral rehabilitation of children with hypodontia and conical tooth shape:A clinical report. Quintessence Int 2006;37:725-730

10. Açıkgöz A, Kademoglu O, Hypohidrotic ectodermal dysplasia with true anodontia of the primary dentition. Quintessence Int 2007;38:853-858. 